\title{
Finding the Thermal Characteristics of a Heat Pipe using Hybrid Nano Fluid
}

\author{
R Rajmohan, B Sivaraman, S Vijayaraj, P Raveendiran
}

\begin{abstract}
In this experiment, work was carried out to infer the thermal characteristics of a heat pipe containing nano fluid inside in it. Various Parameters were considered in this experiment, some of them are inlet temperature at one end, mass flow rate ( $m f r)$ to evaporator section and inclination angle of heat pipe. In this work three numbers of heat pipes were used and hybrid nanofluid of Al2O3 - TiO2 has been used as cooling fluid in all three heat pipes. The thermal efficiency of the usage of hybrid nanofluidic working system is found to be highest and also this makes the system to get worse in terms of thermal resistance. The flow rate of condenser section was modified to the various ratios from 1:1 to 1:3 as that of evaporator section. To find the thermal characteristics of the heat pipe, many experiments have been carried out by considering many operating conditions. Evaluation on the heat pipe effectiveness was made on basis of gravity assistance to the condenser. The better productiveness of heat pipe when using the hybrid nanofluid has attained when $\mathrm{Ch} / \mathrm{Cc}=2$ and $100 \mathrm{LPH}$ for all operating conditions.
\end{abstract}

Index Terms: Effectiveness, Heat pipe, hybrid nanofluid, Heat pipe, Inclination angle, NTU, Thermal performance.

\section{INTRODUCTION}

Heat pipes are having a wide range of applications in many areas for the quick transfer of heat. Heat pipes are sealed tube used to transfer heat by capillary action due to its high thermal conductivity. Heat pipe used as a heat exchanger is a familiar transferring device of heat due to its potency of thermal stability and flexibility in comparison with normal thermal conductors. Mostly heat pipes are drifter or transferring device of heat, as these takes heat of a high concentrated region to a low concentrated region. The heat pipes were very appealing for use as a heat exchangers in many applications. Normally Heat pipes transfer large quantities of heat with less temperature gradient without requiring any kind of external power. A small drop in the temperature is needed to move this heat. Heat pipes are generally sealed and vacuumed and a porous wick like structure for the generation of capillary action with a small amount of cooling fluid was charged in it. Also it can be segregated to three regions which are evaporator, condenser

Revised Manuscript Received on February 05, 2020.

* Correspondence Author

R Rajmohan, is Research scholar in Mechanical Engineering and working in A.V.C College of Engineering at Mayiladuthurai.

B Sivaraman is Professor, Deparment of Mechanical Engineering, Annamalai University, Chidambaram- 608002

S Vijayaraj is Professor in Mechanical Engineering, A.V.C College of Engineering, Mayiladuthurai.

P Raveendiran, is Associate Professor, Deparment of Mechanical Engineering, Alagappa Chettiar Government college of Engineering ,Karaikudi

(C) The Authors. Published by Blue Eyes Intelligence Engineering and Sciences Publication (BEIESP). This is an open access article under the CC BY-NC-ND license (http://creativecommons.org/licenses/by-nc-nd/4.0/) and adiabatic sections. Many parameters like the type of working fluid, pumping pressure, charge ratio, gravity and inclination angle and friction loss will affect the heat transferring capacity of the working fluid[1],[2],[3],[4],[5],[6],[7]. The advantages of heat exchanger with heat pipes are light weight, effective heat recovery, thermal separation between hot and cold fluid, absence of any kind of moving mechanisms and absence of external power required for the operation as mentioned by Dunn and Reay [8]. The working cooling fluid used inside must be chosen wisely to maintain the resistance exerted in the heat pipe. Many researches were conducted in the field of nanofluids as a working fluid in recent days by the researchers. Nanofluids shows a new trends to work as a heat transfer fluids which contains less than or equal to $1 \%$ of materials or tubes having measurement length of about 1-50 $\mathrm{nm}$ were hanged among the conventional fluids used for heat transferring like normal water, alcohol based liquid etc. with high stability[9],[10],[11],[12]. The term "nanofluid" was first called by Choi in Argonne National Laboratory, USA for the nano sized particles[13]. The advantage of the nanofluid than the conventional base fluid is enhancement of its heat transfer performance in addition to the attainment of increased thermal properties. Also, enhancement in the heat transferring characteristics mainly depends on the factors like the shape and dimensions of the particles, thermal properties and the base fluid particles [14]. Dube have investigated the air-to-air thermosyphon heat pipe heat exchanger having water as the cooling fluid of the heat input of about $12 \mathrm{~kW}$ to the evaporator section which is operating below $300^{\circ} \mathrm{C}$ in his research[15]. Busse et al., Rice et al. have found that the gravity-aided heat pipes having a capillary like formation for the protection of the working cooling fluid from the counter flowing vapour in their research[16],[17]. Kemmer, Abhat et al. have reported that the flow in the heat pipes are having low surface tension, but due to the gravity effect the heat pipes can attain the high rate of the heat transfer capacity [18],[19]. Few researchers have reported that the heat transfer performance can be improved with the help of nanofluids like $\mathrm{Ag}$ and $\mathrm{Au}$ in the heat pipes but thermal resistance and temperature gradient was reduced [20]. Based on the above brief review, it has been proposed to design a heat pipe with an heat exchanger to find its thermal characteristics and performance for the effect of different inclination angles of heat-pipes using hybrid nanofluid as a working cooling fluid inside the heat pipe. In this present work, 
GHPHE with $\mathrm{Al}_{2} \mathrm{O}_{3}+\mathrm{TiO} 2$ hybrid nanofluid has been investigated for many mass flow rate and the temperature sensed of hot water and thermal characteristics and performance of the heat exchanger in accordance with the inclination angle of heat pipe along with shell and heat exchanger have been investigated. In this work, three heat pipes have been arranged co-axially and $\in$-NTU method was performed to find its thermal characteristics of the heat exchanger. It has been identified that the implementation of any new technology for the betterment working of any system will increase its durability[21],[22].

\section{GRAVITY HEAT PIPE HEAT EXCHANGER (GHPHE)}

An experimental setup having three number of heat pipes was made for the examination of the thermal characteristics in the present heat pipe heat exchanger. The schematic representation of the experiment setup of GHPHE is displayed in Fig. 1. It contains a shell, heat pipes, and electrical heater with pump, temperature indicator, cooling water pump and thermocouples. The heat pipes used in this work have these main parts: the evaporator section connected to the hot region, the adiabatic section and the condenser section[23].

Fig. 1: Experimental setup of assisted heat pipe exchanger

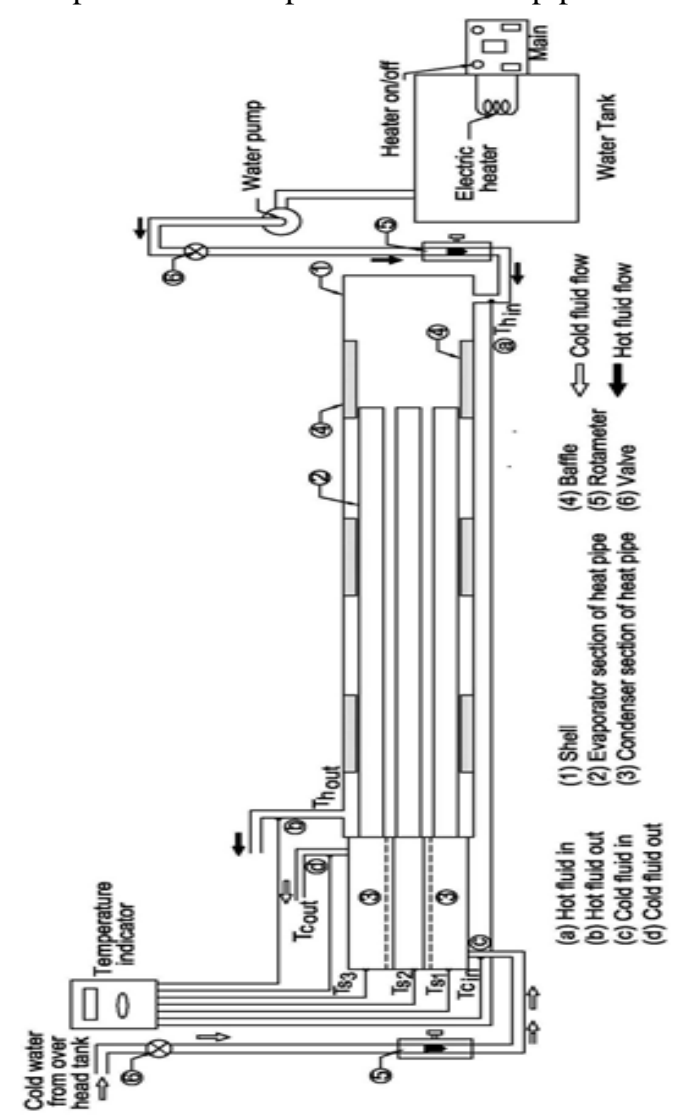

\section{Fig. 1: Schematic representation of assisted heat pipe exchanger}

Two Heaters having the same capacity of $2 \mathrm{~kW}$ was used for the heating of a water tank having 40 litres capacity and the heat from it was drawn from the condenser section by free water circulation in it. The temperature exerted in both hot and cold water has been noted with a digital thermometer. Four RTD was fixed in the setup for calculating the temperature exerted on the hot and cold water. All three heat pipes were charged with hybrid nano fluid which has been used as working flow in GHPHE. The hybrid nanofluid is the combination of $\mathrm{Al}_{2} \mathrm{O}_{3}$ of $30 \%$ of mass of working fluid required for each heat pipe and $\mathrm{T}_{\mathrm{i}} \mathrm{O}_{2}$ of $70 \%$ of mass for the same. The gravitational effect is one of the important factors which was looked attentively for the evaluation of the thermal performance of heat changer and it has been kept at $15^{\circ}$ interval. The influence on the temperature sensed in the hot water having similar mass flow rate throughout the pipe having hot water has been considered for thermal analysis. The laminar flow was considered for all the operating conditions and effectiveness of GHPHE has been calculated by $\in$-NTU method. The details of the working cooling fluid, heat pipe and inclination angle kept on the heat pipe have been tabulated in Table 1.

Table 1: Specification of the working cooling fluid

\begin{tabular}{|c|c|c|}
\hline Wick & Working Fluid & Specification \\
\hline $\begin{array}{c}\text { Stainless steel } \\
\text { mesh }\end{array}$ & $\begin{array}{c}\mathrm{Al}_{2} \mathrm{O}_{2}+\mathrm{THO}_{2} \text { hyborid } \\
\text { nanofluid }\end{array}$ & $\begin{array}{l}\text { - Total length } 1000 \mathrm{~mm} \\
\text { - Wick structure: Mesh no. } 2000,2 \text { layer } \\
\text { - Evaporator section: Copper } 700 \mathrm{~mm} \text { long, } 19 \mathrm{~mm} \\
\text { OD, } 17 \mathrm{~mm} I D \text {. } \\
\text { - Condenser section wate jacket : Copper } 35 \mathrm{~mm} \\
\text { diameter } \\
\text { - Inclination angle: } 15^{\circ}, 30^{\circ}, 45^{\circ} \& 60^{\circ}\end{array}$ \\
\hline
\end{tabular}

\section{RESULTS \& DISCUSSIONS}

In this analysis, the performance of GHPHE depends on the following parameters.

\section{A. Fixed parameters}

- Length and Diameter of Shell,

- Length of condenser and evaporator

- Water as heat transport fluid in shell and condenser side

- Hybrid nanofluid as working fluid in heat pipes.

B. Variable parameter

- Inlet mass flow rate exerted on hot water $\left(\mathrm{T}_{\mathrm{hi}}\right) 20 \mathrm{lph}-100$ lph

- Inlet mass flow rate exerted on cold Water $\left(\mathrm{T}_{\mathrm{ci}}\right) 10 \mathrm{lph}-$ $50 \mathrm{lph}$

- Inlet temperature exerted on the water delivered to the evaporator section: $50^{\circ} \mathrm{C}-70^{\circ} \mathrm{C}$

- Inclination angle $(\Psi): 15^{\circ}-60^{\circ}$

The mass flow rate is a important factor for the determination of the performance and characteristics of heat pipe performance. The mass flow rate exerted on the hot water entering in the inlet of the evaporator section was kept constant for all the sets of experimentation, but the condenser section mass flow rate exerted on the cold water varied in the ratios like 1:1, 1:1.5, 1:2, 1:2.5 and 1.3. For various inclination angles, the effectiveness of heat pipe changes corresponding to the mfr exerted on the water to the evaporator section was shown in the Figs. 2 - 6. From the obtained figures we can conclude that the maximum value of effectiveness of GHPHE was obtained at maximum mfr of hot water to the intake of heat exchanger. Figure. 2 shows the variation in the effectiveness against the mass flow rate for the inclination angle of 0 degree of the heat pipe.

Published By:

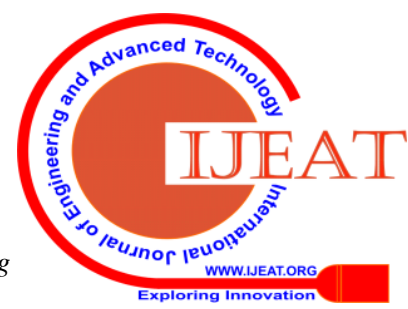


Figure. 3 shows the variation in the effectiveness against the mass flow rate for the inclination angle of 15 degree of the heat pipe. Figure. 4 shows the variation in the effectiveness against the mass flow rate for the inclination angle of 30 degree of the heat pipe. Figure.5 shows the variation in the effectiveness against the mass flow rate for the inclination angle of 45 degree of the heat pipe. Figure. 6 shows the variation in the effectiveness against the mass flow rate for the inclination angle of 60 degree of the heat pipe. In all operating conditions, it is found that the minimum value of effectiveness was obtained when equal amount of hot and cold water are used in shell and condenser side. From the figures it is clear that effectiveness value was increased along with the increase in the mass flow rate upto certain values. After that value the effectiveness value recorded from the heat pipes reduced.

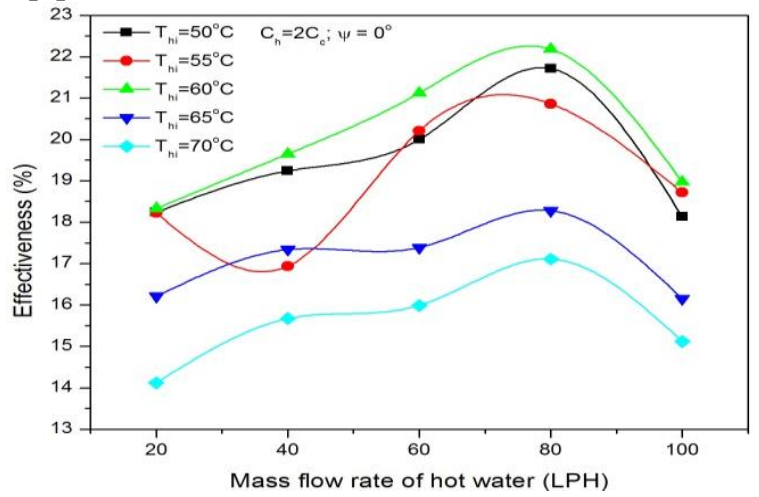

Fig.2: Mass Flow rate Vs Effectiveness

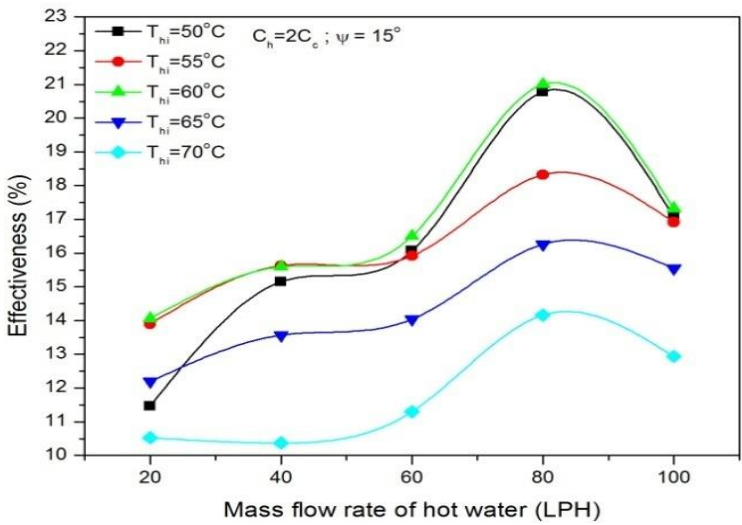

Fig.3: Mass Flow rate Vs Effectiveness

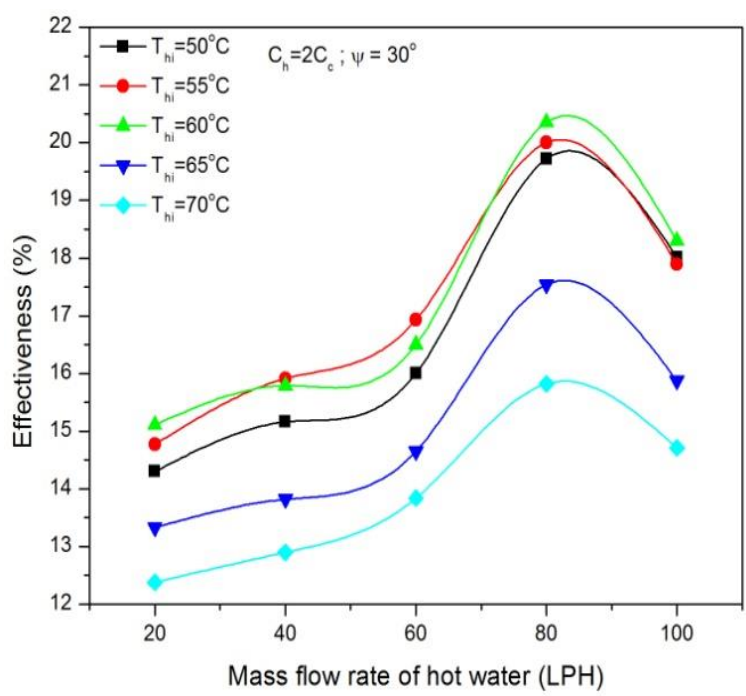

Fig.4: Mass Flow rate Vs Effectiveness

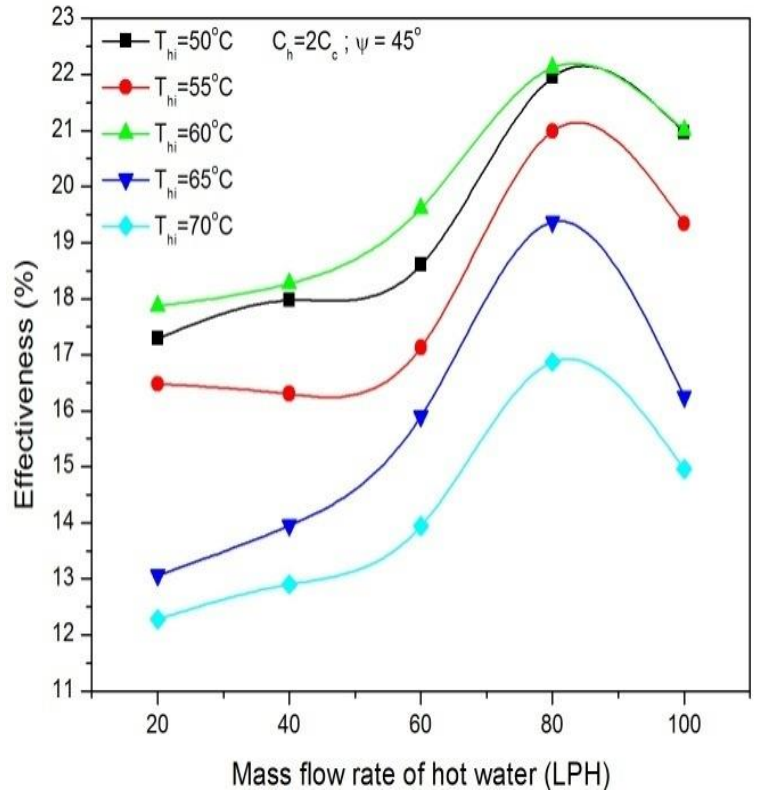

Fig.5: Mass Flow rate Vs Effectiveness

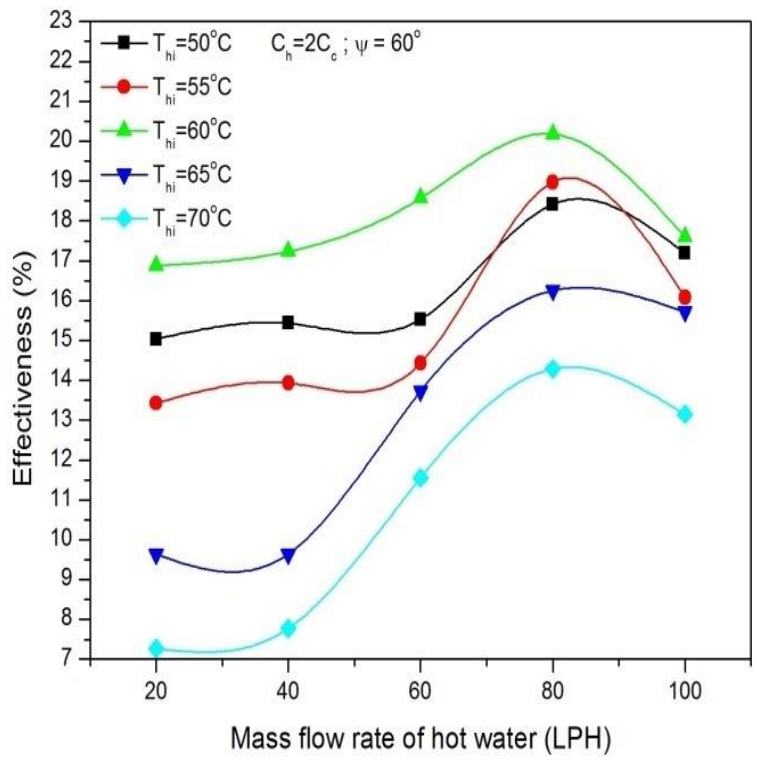

Fig.6: Mass Flow rate Vs Effectiveness

It is clearly observed that the high desirable characteristics of the heat pipe was exerted when the heat capacity rate of hot water is double than amount of heat capacity rate of cold water for all operating conditions. Also the $\mathrm{mfr}$ of $80 \mathrm{lph}$ shows the maximum effectiveness for almost all the inclination angle of heat pipe. This also have a vital role of determining the thermal characteristic of heat pipes.The effectiveness versus heat pipe inclination angle for different inlet temperature of hot water has been shown in Fig. 7. It is inferred $45^{\circ}$ inclination angle is the best position to get high effectiveness from heat pipes for all types of operating. At $45^{\circ}$ inclination of the heat pipe and $80 \mathrm{lph}$ of mfr of hot water at the inlet of heat pipe the optimum thermal characteristics of heat pipe was recorded. 


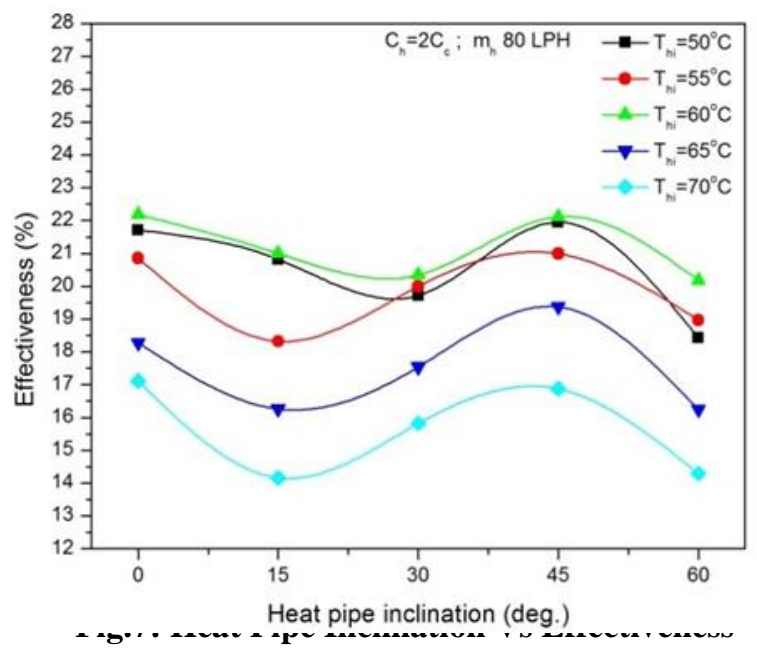

Fig. 8 compares the thermal performance of D.I water and hybrid nano fluid as a cooling liquid inside the heat pipe. For the inclination angle of $0^{\circ}$ and $80 \mathrm{lph} \mathrm{mfr}$ of hot water, the effect due to hot water inlet temperature over effectiveness of heat exchanger for the various values of heat capacity ratio of both D.I water and hybrid nano fluid, shown in Fig. 8. From this figure, it is observed that the optimum value of heat exchanger has been found at $70^{\circ} \mathrm{C}$ and $\mathrm{C}_{h}=2 \mathrm{C}_{\mathrm{c}}$ for all the operating conditions. The effectiveness of heat pipe using the hybrid nanofluid is higher than the heat pipes using DI water as a cooling fluid.

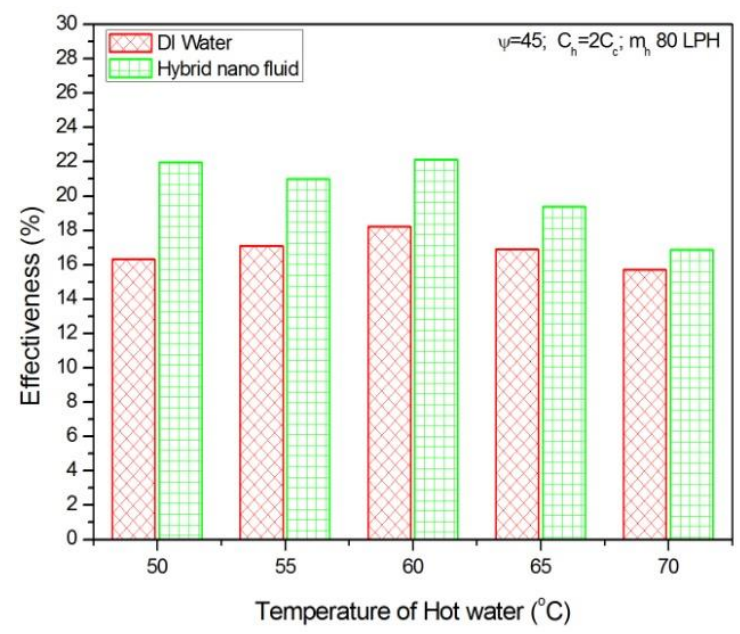

Fig.8: Heat Pipe Inclination Vs Effectiveness

\section{CONCLUSION}

Thus the performance of a heat pipe through the experimental setup was done in this work. The evaluation was carried out with the help of an experimental setup as described above in this paper. The experiment was carried out wisely in order to get the accurate results from the trials. The various parameters were considered properly before doing this experiment and the correct values were noted for each readings. This paper presents the effect of hybrid nanofluid inside the heat pipe was examined on the thermal performance \& characteristics. It also presents the comparative thermal analysis between D.I. water and hybrid nanofluid as cooling medium in the heat pipe. In this GHPHE, it has been found that the nanofluid having significant role in thermal characteristics of a heat pipe. In all set of operating conditions, at lowest mfr of hot water \& minimum intake temperature of hot water at the inlet, the effectiveness of heat exchanger is less. It is concluded that, the optimum effectiveness of GHPHE, has been obtained at mhi=100 lph, $\mathrm{Thi}=70^{\circ} \mathrm{C}$ and $\Psi=45^{\circ}$, when Al2O3 and $\mathrm{TiO} 2$ has been used as working fluid in heat pipe. Thus the performance and characteristics of a heat pipe heat exchanger having the hybrid nano fluid as cooling medium has been evaluated.

\section{REFERENCES}

1. Wang, Zhangyuan \& Zhang, Haopeng \& Chen, Fucheng \& Zheng, Siming \& Huang, Zicong. (2019). Heat Pipe and Loop Heat Pipe Technologies and Their Applications in Solar Systems. 10.1007/978-3-030-17283-1_3.

2. Abdullahi, Bala \& Al-dadah, Raya \& Mahmoud, Sa'ad. (2019). Thermosyphon Heat Pipe Technology. 10.5772/intechopen.85410.

3. Oliveira, Rafael \& Villalva, Sergio \& Filho, Luiz \& Windlin, Fernando \& Alegre, Guilherme \& Santos, Rogério. (2020). Pulsating Heat Pipe Automotive Application. 10.4271/2019-36-0227.

4. Mizuta, Kei \& Tsuruta, Katsuya \& Kotani, Toshiaki \& Ohsawa, Kenji. (2013). Heat pipe and circuit board with a heat pipe function.

5. Hu, Kaibo \& Su, Lizheng \& Liu, Jipeng. (2020). Structure Optimization of the Heat-Concentrated Radiator with Heat-Pipe. 10.1007/978-981-32-9441-7_28.

6. Karimi G., Culham J.R., "Review and Assessment of Pulsating Heat Pipe Mechanism for High Heat Flux Electronic Cooling”, 2004 Inter Society Conference on Thermal Phenomena, pp 52-58

7. Cheverda, Viacheslav \& Ronshin, Fedor. (2019). Water pulsating heat pipe. EPJ Web of Conferences. 196. 00043. 10.1051/epjconf/201919600043.

8. P.D. Dunn, D.A. Reay (1994) Heat Pipes, third ed., Pergamon Press.

9. Zohuri, Bahman. (2019). Heat Pipe Manufacturing. 10.1007/978-3-030-05882-1_10.

10.Ong, K. \& Tan, M. \& Chong, K.. (2019). Thermal performance of sintered heat pipes. AIP Conference Proceedings. 2157. 020058. 10.1063/1.5126593.

11.Vijayasarathy, B \& Kumar, Dinesh \& Vijayakrishnan, K \& Kumar, Ajith \& Kotebavi, Vinod. (2019). Solar distillation using heat pipes and heat exchanger. IOP Conference Series: Materials Science and Engineering. 577. 012025. 10.1088/1757-899X/577/1/012025.

12.Mekcem, Maroua. (2018). Nanofluids and heat pipe limitations. $\begin{array}{lllr}\text { Academic } \quad \text { Perspective } \quad \text { Procedia. } & 1 . & \text { 298-304. }\end{array}$ 10.33793/acperpro.01.01.58

13.Cheverda, Vyacheslav \& Ronshin, Fedor. (2019). Experimental study of heat transfer in a heat pipe. Journal of Physics: Conference Series. 1369. 012053. 10.1088/1742-6596/1369/1/012053.

14.Sharma, Megha \& Sikarwar, Basant. (2019). Flow and heat transfer of fluid in a pulsating heat pipe. Journal of Physics: Conference Series. 1369. 012019. 10.1088/1742-6596/1369/1/012019.

15.V. Dube, (1996), Design construction and testing of a thermosyphon heat exchanger under medium temperature heat recovery, Proceeding of the $5^{\text {th }}$ International heat-pipe symposium, Melbourne Australia, pp.273-279.

16.C.A. Busse and J.E. Kemme, "dry-out phenomena in gravity -assisted heat pipes with capillary flow”, Int, J. Heat Mass Transfer, 23, 643-654 (1980).

17.G. rice and E. Azad, "Dynamic Characteristics of Heat pipes", Proceedings of 2nd Int. Heat Pipe Conf., Bologna, Italy, ESA, The Netherlands, 153-164 91976).

18.J.E. Kemme, "Vapour flow considerations in conventional and gravity-assist heat pipes", Proceedings, 2nd Int, Heat Pipe conf., Bologna, Italy, ESA, The Netherlands, 11-22 (1976).

19.A. Abhat and H. Nguyenchi, "Investigation of Performance of gravity assisted copper-water heat pipes", Proceeding, 2nd Int, Heat-Pipe conf., Bologna, Italy, ESA, The Netherlands, 23-26 (1976).

20.Zhengping, Xi \& Huiping, Tang \& Jilei, Zhu \& Jichang, Liao. (2006). Heat pipes and porous metals used in heat pipes. RARE METAL MATERIALS AND ENGINEERING. 35. 418-422. 
21.Elavarasan.G Kannan M, Thiagarajan.L, Karthikeyan.D. Performance Characteristics \& Emission Analysis Of Mustard Oil Based Biodiesel In CI Engine Using Exhaust Gas Recirculation. INTERNATIONAL JOURNAL OF SCIENTIFIC \& TECHNOLOGY RESEARCH VOLUME 2019;8(8):878-82.

22.Elavarasan G, Kannan M, Karthikeyan D. Experimental Analysis to find the optimum Specific Fuel Consumption for Driver's Intimation. International Journal of Recent Technology and Engineering 2019;8(2):452-6.

23.Madhav, H \& Rauhavendra, Venkata \& Kumar, Pramod \& Ambirajan, Amrit. (2019). Analytical Model for a Cylindrical Heat Pipe. 251-260. 10.1109/ITHERM.2019.8757290.

\section{AUTHORS PROFILE}

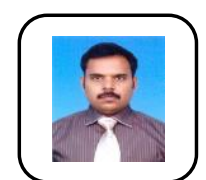

R Rajmohan is a Research scholar in the Mechanical Engineering department of A.V.C College of Engineering which is located Mayiladuthurai, Tamil Nadu.

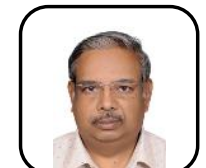

B Sivaraman is working as a Professor in the Deparment of Mechanical Engineering, FEAT, of the premier university of India Annamalai University, Annamalainagar. Author has pulished various papers in the web of science indexed journal. Also he is the head of the department of Mechanical Engineering of Annamalai University.

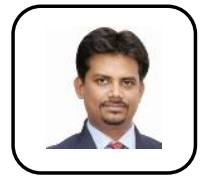

S Vijayaraj is a Professor working in the Department of Mechanical Engineering of A.V.C College of Engineering which is locatd in Mayiladuthurai, Tamil Nadu.

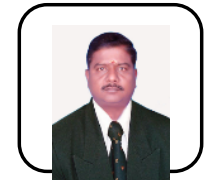

P Raveendiran is a Associate Professor working in the Deparment of Mechanical Engineering of Alagappa Chettiar Government college of Engineering which is located in Karaikudi. 